\title{
APOL 1 and kidney disease
}

\author{
Carlos E. Duran ${ }^{1 \star}$, Fredy Lozano ${ }^{2}$ \\ ${ }^{1}$ Department of Nephrology, Fundacion Valle del Lili, Cali, Colombia \\ - Icesi University, Cali, Colombia \\ ${ }^{2}$ Nephrology Fellow, Universidad del Valle, Cali Colombia
}

\author{
${ }^{*}$ Corresponding author \\ Carlos E. Duran, Department of Nephrology, Fundacion Valle del Lili, \\ Cali, Colombia - Icesi University, Cali, Colombia
}

Submitted: 23 Aug 2020; Accepted: 29 Aug 2020; Published: 11 Sept 2020

\begin{abstract}
Background
APOL1 gene variants have been described to offer higher risk to develop hypertension, collapsing nephropthy and end stage kidney disease. The prevalence of mutations of the APOL1 gene among afro-descendant patients with chronic kidney disease for the G1 and the G2 variants can be of 20-22\% and 13-15\%, respectively [1].

Moreover this can be a greater problem in afrodescendante based populations, for instance, in Colombia, the genetic background of the populations was shaped by different levels of admixture between Natives, European, and Africans. Approximately 35.363 patients have diagnosed chronic kidney disease and according to population studies, 10.4\% of these patients are Afro-descendant, and these frecuency can be as high as $90 \%$ in some áreas of this country.

Given the importance that accounts on this area in the last years, the purpose of this review is to highlight the majors improvements in the understanding on the biology, pathophysiology and kidney disease development on patients affected with this genetic high risk variants.
\end{abstract}

Keywords: APOL1, kidney disease, kidney transplantation

\section{APOL1: Biological Aspects}

APOL1 is a member of the APOL gene family encoded on chromosome 22, this gene encodes a protein called apolipoprotein L1 (apol1). They are found in most animals but are only expressed and functional in humans, gorillas and baboons [2].

The apol1 protein is present in two states; circulating and attached to the cell. Circulating apol1 is bound to HDL particles, secreted in the liver and is increased during periods of inflammation. Apol1 associated with tissues is found distributed in many organs, with a special importance in the renal tissue specifically in the podocyte and proximal tubules $[3,4]$.

The circulating fraction of apol1 imparts resistance to trypanosomiasis by rapidly lysing trypanosomes, this circulating fraction of apol1 is part of the so-called human trypanolitic factor (TLF). There are two types of TLF; TLF1 is made up of apoll, a primate-specific haptoglobin-related protein (Hpr), hemoglobin, paraoxonase, apoA-II, and is the main factor associated with tripanosome lysis. On the other hand, TLF 2 is a larger complex but not very well characterized [5].

The mechanisms of death of trypanosomal cells related to the APOL1 gene are not yet clear, however, a gene organization was found in three domains; a pore-forming domain, a $\mathrm{pH}$-sensitive membrane targeting domain, and a serum resistance-associated interaction domain (SRA) $[3,4]$.

In order for the trypanosome to be lysed, the TLF1 is endocytosed by the parasite through a specific receptor of the haptoglobin-hemoglobin complex, subsequently it is taken to the lysosomes and the progressive acidification in this environment produces conformational changes of the APOL1 address domain. The apol 1 particle is released from the complex, causing the formation of an ion channel in the lysosome membrane that leads to an influx of chloride, producing osmotic stress, cellular and parasite edema $[2,4,6]$.

However, the Gambiense and Rhodesiense trypanosoma have developed mechanisms of resistance to toxicity mediated by APOL1, through the development of the glypoprotein SRA that binds to apol1, canceling its toxicity and through a specific glycoprotein (TgsGP) that forms hydrophobic $\beta$ sheets in the membrane, preventing the formation of pores [7].

As a consequence of this, the APOL 1 sequences evolved positive selection, forming the G1 and G2 mutations, which is the gene domain that interacts with SRA, allowing to restore the toxicity of APOL 1 to Tb rhodesiense, these alleles have been described to be associated with kidney disease $[2,4]$. 
These G1 and G2 mutations encodes changes in the APOL1 binding domain. The G1 mutation is a two amino acid substitution mutation ( $\mathrm{S} 342 \mathrm{G}$ and $\mathrm{I} 384 \mathrm{M}$ ) and the $\mathrm{G} 2$ mutation is a six base pair deletion mutation. Both mutations are exclusive and are not evident on the same chromosome [3].

These Genetic variants in the APOL1 gene, are found only in people of African descent. Initial epidemiological studies describe a high frequency in sub-Saharan Africa populations in relation to the association that this mutation confers against trypanosome infection. Several studies have suggested the importance of APOL1 in innate immunity, since the APOL1 genes are positively regulated by inflammatory cytokines including interferon gamma and tumor necrosis factor alpha; this is associated with its role in other infections such as Leismaniasis and the restriction of HIV replication in macrophages [2].

\section{Apol1 Associated Kidney Disease}

Studies have found that Afroamerican patients have a high burden of chronic kidney disease, even after adjusting other risk variables, therefor a high genetic burden associated with frequent nephropathies was suspected in this ethnic group, especially focal segmental glomerulosclerosis (FSGS) and HIV- associated nephropathy (HIVAN) [8].

In 2010, through the 1000 Genomes Project, a very strong association was established between the APOL1 alleles, focal segmental glomerulosclerosis and chronic kidney disease attributed to hypertension, which is why several clinical trials have been initiated based on the possible pathophysiological mechanisms and outcomes for the development of kidney disease $[9,10]$.

The presence of APOL1 gene variants are found in homozygous (G1 / G1 or G2 / G2), heterozygous (G1 / G0 or G2 / G0) or heterozygous compound forms (G1 / G2). Patients with homozygous and heterozygous compound genotypes (with presence of two risk alleles) have a higher risk of kidney disease, this association was found in case-control studies since the probability of developing glomerulosclerosis (FSGS) was 19 times and HIV- associated nephropathy (HIVAN) was 89 times in patients with HIV respectively in the groups with high-risk genotypes. [11, 12].

After finding this association, several groups around the world were interested in finding models that explained the mechanisms of kidney damage associated with this gene. Multiple studies have been carried out with kidney cell lines and animal models such as drosophila and rat models, however, they are still not totally clear [4].

As mentioned previously, APOL 1 has a circulating form and another form, associated with tissues. Initially an attempt was made to determine whether circulating APOL1 was responsible for the pathophysiological mechanisms of kidney damage, but evidence has demostrated that the circulating form of apolipoprotein 1 levels were not associated with the high-risk APOL1 genotype, and the plasma levels were not associated with the development of chronic kidney disease or with the severity of the disease [13]. Therefor there has been a special focus on the APOL 1 associated with tissues form. Initially it was determined that APOL1 was found in renal podocytes, glomerular endothelial cells and renal tubule cells on renal cryo-sections, but not in mesangial cells [10].

In the first rat models, it was possible to document that the expression of G1 or G2 mutation variants in podocytes induced albuminuria. Albuminuria was a consequence of podocyte foot process effacement that is characteristic of patients with APOL1associated kidney disease [14].

A study based on a cell culture model was able to demonstrate a possible cell toxicity pathway based on the theory that APOL1, forms selective pores in cell membranes by increasing potassium outflow currents, thus producing intracellular potassium depletion. This decrease in intracellular potassium resulted in activation of a stress-activated protein kinases (SAPK), p38 and JNK, inducing cytotoxicity and cell death [15].

Another study demonstrated a second possible mechanism, where APOL1 was associated with mitochondrial dysfunction and a transforming growth factor beta (TGFB) expression, inducing the activation of SAPK, p38 and JNK. Mitochondrial dysfunction was evidenced by a decrease in respiratory capacity and a decrease in the rate of mitochondrial oxygen consumption. Characteristically, this deterioration in mitochondrial function occurred before the decrease in intracellular potassium, described in previous studies [16].

Other proposed mechanisms of podocyte damage suggest that the G2 risk variant activates the NLRP3 inflammasome. Emerging data also suggest that risk variants, through extended doublestranded RNA structures, activate protein kinase R, leading to decreased protein synthesis and promoting cell death [11].

Some studies have shown that renal risk variants are related to defective autophagy processes associated with altered intracellular traffic. Autophagy is necessary to eliminate damaged mitochondria and a disordered mechanism would induce death of the podocyte, given the absence of its proliferation [9]. All these mechanisms and even others related to mitochondrial dysfunction demonstrates an alteration in the intracellular microenvironment of the podocyte, however, despite the high-risk genotype, not all patients develop renal injury associated with APOL1; approximately $20 \%$ of Afrodescendants develop nephropathy, and it is here that researchers propose the second HIT theory [10]. 
These environmental factors have been documented in several studies and are especially associated with the development of collapsing glomerulopathy; within these we have viral entities such as HIV, CMV, EBV and recently SARS Cov2, systemic conditions such as systemic lupus erythematosus (SLE), hemophagocytic syndrome, renal allograft; genetic diseases such as STING associated vasculopathy with onset in infancy (SAVI) and the application of interferon. In a striking and very interesting way is the common pathway of interferon activation in the pathophysiological development of each of these etiologies [17].

The application of interferon has been described as increasing APOL1 levels in kidney tissues and its association with the development of collapsing glomerulopathy in patients with a highrisk genotype. In these studies, it has been proposed as a cause of the disease, given the close temporality relationship between the administration of interferon and the development of glomerulopathy. Despite these findings, there is still no clarity of the pathophysiological mechanism that explains this relationship [18].

In patients with HIV, the role of interferon has been studied extensively, finding that the type of interferon gamma is the one that has the most power to boost the expression of the APOL1 gene, this is achieved by activating STAT 3, occupying the promoter sites of APOL1 [19]. Once this second HIT occurs, the processes of autophagy and alteration of mitochondrial function in podocytes and the development of collapsing glomerulopathy take place [17].

Other associations with circulating factors have been reported in young patients and the development of recurrent post-transplant FSGS, among these the most studied is suPAR. Some interactions of suPAR with the high-risk APOL1 genotype have been described and it seems that they increase the risk of developing chronic kidney disease, however more studies are required to support these findings [10].

\section{Characteristics of APOL1- associated kidney disease}

There are several forms of presentation in APOL1-associated nephropathy, ranging from intense proteinuria with characteristics of nephrotic syndrome associated with deterioration of renal function, to a presentation of subnephrotic proteinuria, with slow deterioration of its GFR, in general being diagnosed erroneously as a hypertensive nephrosclerosis [11].

There are three diseases most frequently associated in order of probability: HIVAN, focal glomerulosclerosis, and arterial hypertension attributed to CKD. As previously mentioned, patients with a high-risk genotype are 17 to 19 times more likely to develop FSGS and 89 times more likely to develop HIVAN in patients with HIV. In a study conducted in the USA of patients with FSGS without HIV, it was found that $72 \%$ of patients had high-risk genotypes compared to $13 \%$ of patients in the general African-
American population, however this probability varies depending on the study that reports them, with some biases given given the small sample size of most studies [19].

Regarding kidney biopsy, most of these patients who are taken to kidney biopsy were those with greater deterioration in renal function or nephrotic proteinuria, so these findings could only be correlated with advanced stages of the disease. Among the histopathological findings, included a collapsing focal segmental glomerulosclerosis and NOS, interstitial fibrosis, tubular atrophy with findings of a thyroidization pattern of the tubules, and no severe arteriosclerosis were found in these patients. Furthermore, in later studies, they found a certain degree of correlation with thyroid-type tubular atrophy, higher degrees of interstitial fibrosis and higher percentages of microcystic tubular dilation in patients with 2 (rather than 0 or 1) APOL1 renal risk variants [11].

Hypertension is another frequent finding in patients with a renal risk variant of APOL1. In a published review from the Mount Sinai biobank, the frequency of risk variants in African American hypertensive patients was analyzed according to APOL1, finding that 14 to $16 \%$ of patients were homozygous for renal risk variants. Additionally, it was found that these patients had a diagnosis 2 to 5 years earlier than the other population without risk variant associated with APOL1. The deterioration of renal function was after 10 years of diagnosis of hypertension in these patients, which is why a possible effect on blood pressure has been suggested rather than with the development of CKD [19].

In a pubished study were we aim to assess the frequency of APOL1 variants $\mathrm{G} 1$ and $\mathrm{G} 2$ in Afro-descendant patients with ESRD treated at la Fundacion Valle del Lili University Hospital in Cali, Colombia, we found that $37 \%$ of patients had $A P O L 1$ high-risk status in which two alleles were in homozygous $(\mathrm{G} 1 / \mathrm{G} 1=21$ and $\mathrm{G} 2 / \mathrm{G} 2=3)$ or compound heterozygote $(\mathrm{G} 1 / \mathrm{G} 2=14)$ form and $63 \%$ had $A P O L 1$ low-risk status [21].

This findings hightened the importance of the pathology in certain áreas were the frecuency of afrodescendant patients is high and highlights the efforts that need to be done in this área.

\section{APOL1 and Kidney Transplantation}

The first observations were made about the short survival of the renal allograft from Afro-descendant patients compared to that from European patients. Subsequently, it was shown that the donor APOL1 genotype contributes significantly to a decrease in renal allograft survival. The APOL1 genotype of the recipient had no association [22].

In a subsequent study of 1153 kidney donors, a multivariate analysis was performed taking into account risk variables such as the age of the donor and recipient, cold ischemia time, HLA 
compatibility, PRA, and donation with extended criteria, finding a longer survival reduction of the renal allograft in APOL1 kidney recipients with 2 renal risk variants (risk index [HR], 2.00; P = 0.03 ), this associated with the older donor age and the youngest recipient age negatively affected allograft survival [23].

Regarding living donor kidney transplantation, APOL1 has been shown to be involved in the development of progressive GEFS with allograft failure in recipients and with ESKD in previously healthy African-American donors. These findings raise the question whether it is necessary to perform genotyping of donors prior to transplantation [11]. Some tests have been carried out to modify the scores assigned to donor used in other countries (KDPI), finding that replacing race with APOL1 genotype would more accurately quantify the risk associated with the kidneys of African American donors, and would improve the match between donor quality and recipient patient's need.

There is currently a large scale study regarding these questions, named under the APOLLO network, APOL1 long-term kidney transplantation outcomes network, and this results may guide, decisions on the assessment of quality in deceased donor kidneys.

\section{Conclusions}

Apol1, can represent a high burden of kidney disease, that can be manifested in different ways from hypertension to end stage kidney disease, and heightens the importance on prevention public policies on afrodescendant population. Moreover, is important to make more studies, because the admixture between ratial population can be high in some areas, and genetic variants can be important.

Meanwhile, efforts must be done on the understanding of the biology and pathophysiology of the disease, aming therapeutic targets to control and delay kidney disease. There is a lot of field for basic research and clinical developments on this area, not just for kidney disease but also on transplant field, where we are sure that results of the APOLLO study will guide use of APOL1 genotypic data to improve the assessment of quality in deceased-donor kidneys increasing the number of transplanted kidneys, reducing rates of discard, and improve the safety of living-kidney donation.

This review highlights major advances on the understanding of the pathophisiology of apol1 kidney disease. There are new research áreas with important issues that need thourough research, like that compelling interferón association on the pathophisiology of disease. More studies have to be done, to understand the rol of this protein on the development of apoll disease on a second hit theory.

\section{References}

1. Limou S, Nelson GW, Kopp JB, Winkler CA (2014) APOL1 Kidney Risk Alleles: Population Genetics and Disease Associations. Adv Chronic Kidney Dis 21: 426-433.
2. Limou S, Dummer PD, Nelson GW, Kopp JB, Winkler CA, et al. (2015) APOL1 toxin, innate immunity, and kidney injury. Kidney Int 88: 28-34.

3. Friedman DJ (2020) A Brief History of APOL1_A Gene Evolving. Semin Nephrol 37: 508-513.

4. Bruggeman LA, Madhavan S, Sedor JR, Western C (2018) The cell biology of APOL1 37: 538-545.

5. Raper J, Fung R, Ghiso J, Nussenzweig V, Tomlinson S, et al. (1999) Characterization of a novel trypanosome lytic factor from human serum. Infect Immun 67: 1910-1916.

6. Molina Portela MDP, Lugli EB, Recio-Pinto E, Raper J (2005) Trypanosome lytic factor, a subclass of high-density lipoprotein, forms cation-selective pores in membranes. Mol Biochem Parasitol 144: 218-226.

7. Uzureau P, Uzureau S, Lecordier L, Fontaine F, Tebabi P, et al. (2013) Mechanism of Trypanosoma brucei gambiense resistance to human serum. Nature 501: 430-434.

8. Freedman BI, Limou S, Ma L, Kopp JB (2018) APOL1 -Associated Nephropathy: A Key Contributor to Racial Disparities in CKD. Am J Kidney Dis 72: 8-16.

9. CDC Chronic Kidney Disease in the United States (2019) Cdc $1: 1-6$.

10. Divers J, Freedman BI, Carolina N, Carolina N (2020) Mechanisms of injury in APOL1-associated kidney disease. Transplantation 103: 487-492.

11. Mirza A, Kowalewska J, Mccune T, Bruggeman LA, Madhavan S, et al. (2020) APOLl-Associated Nephropathy: A Key Contributor to Racial Disparities in CKD. Semin Nephrol 37: 530-537.

12. Kopp JB, Nelson GW, Sampath K, Johnson RC, Genovese G, et al. (2011) APOL1 genetic variants in focal segmental glomerulosclerosis and HIV-associated nephropathy. J Am Soc Nephrol 22: 2129-2137.

13. Bruggeman LA, O'Toole JF, Ross MD, Madhavan SM, Smurzynski M, et al. (2014) Plasma apolipoprotein L1 levels do not correlate with CKD. J Am Soc Nephrol 25: 634-644.

14. Beckerman P, Susztak K (2020) APOL1: The Balance Imposed by Infection, Selection, and Kidney Disease. Trends Mol Med 24: 682-695.

15. Olabisi OA, Zhang JY, VerPlank L, Zahler N, DiBartolo S, et al. (2016) APOL1 kidney disease risk variants cause cytotoxicity by depleting cellular potassium and inducing stress-activated protein kinases. Proc Natl Acad Sci 113: 830-837.

16. Ma L, Chou JW, Snipes JA, Bharadwaj MS, Craddock AL, et al. (2017) APOL1 renal-risk variants induce mitochondrial dysfunction. J Am Soc Nephrol 28: 1093-1105.

17. Velez JCQ, Caza T, Larsen CP (2020) COVAN is the new HIVAN: the re-emergence of collapsing glomerulopathy with COVID-19. Nat Rev Nephrol 1-3. 
18. Pengse Po, Erin Delaney, Howard Gamper, Miklos SzantiKis, Lee Speight, et al. (2017) Innate immunity pathways regulate the nephropathy gene Apolipoprotein L1. Physiol Behav 176: 139-148.

19. Kopp J, Jurgen H, Winkler CA (2017) APOL1 renal risk variants: Fertile soil for HIV-associated nephropathy. Semin Nephrol 176: 139-148.

20. Manolio TA, Chisholm RL, Roden DM, Denny JC, Kenny EE, et al. (2020) Apolipoprotein L1 Variants and Blood Pressure Traits in African Americans. J Am Coll Cardiol 69: 1564-1574.
21. Duran CE, Ramírez A, Posada JG, Schweineberg J, Mesa L, et al. (2019) Prevalence of APOL1 Risk Variants in AfroDescendant Patients with Chronic Kidney Disease in a Latin American Country. Int J Nephrol.

22. Freedman BI, Locke JE, Reeves-daniel AM, Julian BA (2020) Apolipoprotein L1 Gene Effects on Kidney Transplantation. Semin Nephrol 37: 530-537.

23. Freedman BI, Pastan SO, Israni AK, Julian BA, Gautreaux $\mathrm{MD}$, et al. (2017) APOL1 genotype and kidney transplantation outcomes from deceased African American donors Barry 100: 194-202.
Copyright: (C2020 Carlos E. Duran, et al. This is an open-access article distributed under the terms of the Creative Commons Attribution License, which permits unrestricted use, distribution, and reproduction in any medium, provided the original author and source are credited. 